\title{
PANDA detector system
}

\author{
H. $\operatorname{Orth}^{a}$ \\ ${ }^{a}$ Gesellschaft für Schwerionenforschung, Planckstrasse 1, 64291 Darmstadt, Germany
}

\section{Introduction}

The PANDA detector system is a universal instrument to study fundamental questions of hadron and nuclear physics in interactions of antiprotons with protons and nuclei $[1,2,3]$. Charmonium spectroscopy, gluonic excitations and the physics of strange and charm quarks will be accessible with unprecedented accuracy thereby allowing measurements of strongly interacting systems with high precision.

The proposed PANDA detector is a state-of-the-art internal target detector to be installed at the high-energy storage ring (HESR) at the Facility for Antiproton and Ion Research (FAIR). It will allow the detection and identification of neutral and charged particles generated within the relevant angular and energy range. This task will be shared by the combination of a central and a forward spectrometer of modular design which both are optimized for the specific kinematics of the antiproton-nucleon annihilation process.

The detector design incorporates the most recent technologies in order to reach the required performance criteria with regard to mass, momentum and energy resolution, hit resolution, particle identification, and solid angle coverage. The combination of the highquality antiproton beam and the detector system of almost full hermiticity provides a powerful and unique facility for hadron physics.

\section{Physics motivation}

Experimentally, studies of hadron structure can be performed with different probes such as electron, pion, kaon, proton or antiproton beams, each of which have its specific advantages. In antiproton-proton annihilation, particles with gluonic degrees of freedom as well as particle-antiparticle pairs are copiously produced, allowing spectroscopic studies with unprecedented statistics and precision. Antiprotons of 1.5 to $15 \mathrm{GeV} / \mathrm{c}$ momentum will therefore be an excellent tool to address the open problems mentioned below. The following experiments are foreseen:

Charmonium spectroscopy: precision measurements of mass, width, decay branches of all charmonium states, especially for extracting information on the quark confining potential. The unequaled resolution in the $\bar{p} p$ formation process and small systematic uncertainties give the unique opportunity to improve dramatically our knowledge which cannot be 
achieved elsewhere.

Exotic hadrons: firm establishment of the QCD-predicted gluonic excitations (charmed hybrids, glueballs) in the charmonium mass range $(3-5 \mathrm{GeV} / \mathrm{c})$ using high statistics in combination with sophisticated spin-parity analysis in fully exclusive measurements

In nuclear medium modifications: search for modifications of meson properties in the nuclear medium, and their possible relationship to the partial restoration of chiral symmetry for light quarks. Particular emphasis is put on mesons with open and hidden-charm, extending ongoing studies in the light quark sector to heavy quarks and aiming for new information on contributions of the gluon dynamics to hadron masses.

Hypernuclear physics: precision $\gamma$-ray spectroscopy of single and double hypernuclei for extracting information on their structure and on the hyperon-nucleon and hyperonhyperon potential.

As soon as the HESR facility reaches the full design luminosity further physics opportunities will open up like: Extraction of generalized parton distributions from $\bar{p} p$ annihilation, D meson decay spectroscopy (rare leptonic and hadronic decays), and Search for CP violation in the charm and strangeness sector (D meson decays, $\Lambda \bar{\Lambda}$ system).

\section{Antiproton Storage Ring (HESR)}

Antiprotons are being produced by bombarding $30 \mathrm{GeV}$ protons from the SIS 100 synchrotron on a iridium target using a magnetic horn as collector lens. They are transferred into the CR-complex consisting of two storage rings, one acting as collector, bunch rotator and pre-cooler, the second as accumulator for stacking and cooling the antiprotons at $3 \mathrm{GeV}$ energy. The production rate of properly conditioned antiprotons should be $2 \times 10^{7}$ per second. After an accumulation time of one hour about $10^{11}$ antiprotons will be collected and directly transferred into the HESR (see fig.1). Subsequently further electron/stochastic cooling is applied and, using the synchrotron mode of HESR, the antiprotons are accelerated / decelerated to the desired momentum. The momentum range is between 1.5 and $15 \mathrm{GeV} / \mathrm{c}$, the momentum bite after cooling $\Delta \mathrm{p} / \mathrm{p}=10^{-4}$ to $10^{-5}$. The number of initially stored antiprotons may vary from $10^{10}$ to $5 \times 10^{11}$ and a maximum luminosity of $2 \times 10^{32} \mathrm{~cm}^{-2} \mathrm{~s}^{-1}$ is envisaged.

The Panda detector is located in one of straight sections of HESR, where beam optics is made dispersionless and a low $\beta$-section is foreseen. The $\beta$-function at the target point must be less than $1 \mathrm{~m}$ for achieving a minimal focal spot. Transverse and/or longitudinal emittances should be as small as possible.

\section{PANDA detector}

The PANDA detector (see fig.2) comprises two magnetic spectrometer configurations. A solenoidal magnetic field surrounds the target called target-spectrometer (TS) and a large aperture dipole magnet accepts the reaction product in a 5 degree cone towards forward direction. This part is named forward-spectrometer(FS). From the point of view of particle identification the full $4 \pi$ acceptance of PANDA is sectioned into three parts. From backward to $22^{\circ}$ forward direction the barrel-type detectors are at work, from $22^{\circ}$ 


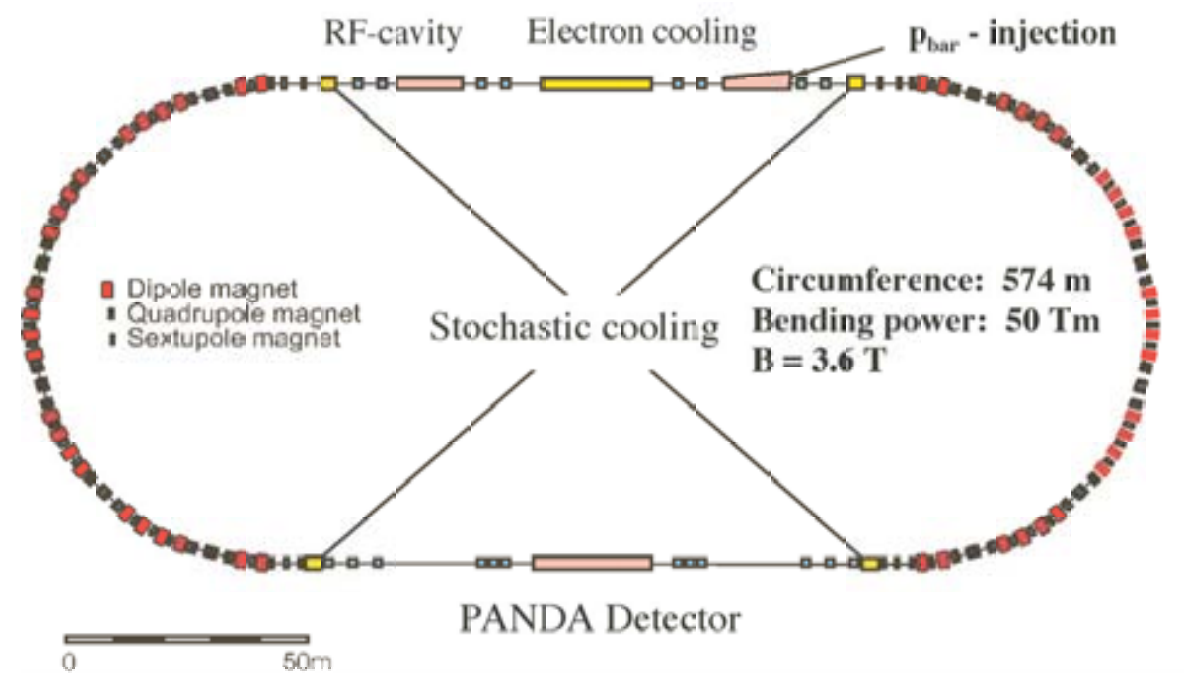

Figure 1: High energy storage ring for antiprotons (HESR)

to $5^{\circ}$ degrees a forward detector section is installed within the solenoidal field of the TS and from $5^{\circ}$ to almost $0^{\circ}$ all information comes from the forward spectrometer (FS).

\subsection{Target spectrometer}

\subsubsection{Internal Targets and interaction section}

Experiments with internal targets in a storage ring used gas, cluster-jet or wire targets. A more recent development is the pellet target presently set up in the Celsius storage ring. The basic geometrical properties considered for the internal target in experiments with close to $4 \pi$ acceptance are the diameter of the beam pipe and the diameter of the target mandrel. For PANDA both pipes are only $20 \mathrm{~mm}$ wide at the intersection. The target mandrel opens up gradually reaching $100 \mathrm{~mm}$ outside the calorimeter and $350 \mathrm{~mm}$ through the iron yoke. The beam pipe opens up in backward direction to 140 $\mathrm{mm}$ diameter, in order to provide sufficient pumping power. In downstream direction it stays at $20 \mathrm{~mm}$ diameter until the end of the vertex tracker. Distributed pumping via NEG coated surfaces is considered everywhere within the intersection section. This setup will allow different internal targets. Currently, both a pellet target and cluster-jet target are being developed to fit inside the target chamber. Given a stored number of $10^{11}$ antiprotons in the HESR a target density of $3 \times 10^{15}$ hydrogen atoms per $\mathrm{cm}^{2}$ is required to reach the design luminosity. 


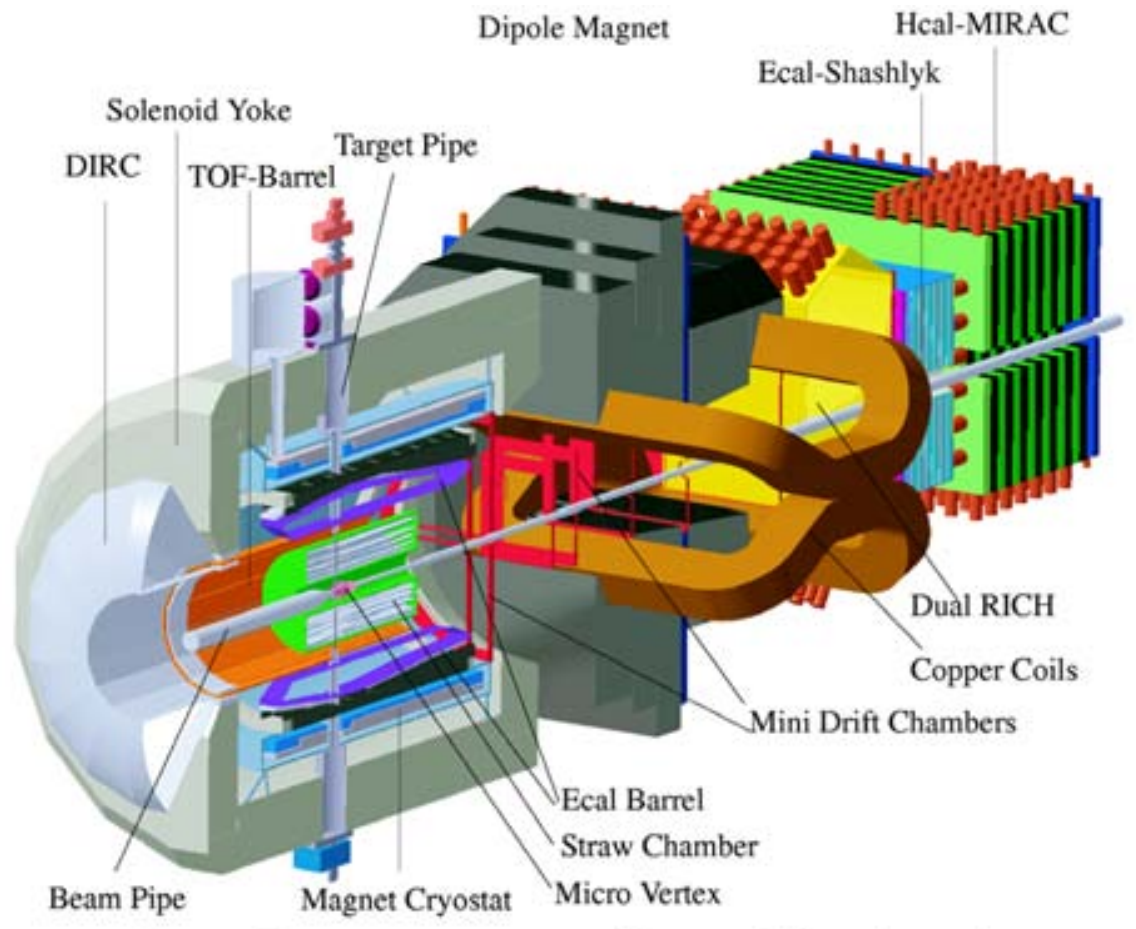

\section{Target Spectrometer Forward Spectrometer}

Figure 2: PANDA Detector

\subsubsection{Cluster-Jet Target.}

To fulfill this demand with a cluster-jet target requires nozzle developments leading to a density increase of one order of magnitude compared to what has presently been achieved. In addition, due to detector constraints, the distance between the cluster-jet nozzle and the target has to be increased compared to other applications. The size of the target region will be given by the lateral spread of hydrogen clusters, which, when optimized, should stay less than $10 \mathrm{~mm}$. The great advantage of cluster targets is the homogeneous density profile and the possibility to focus the antiproton beam at highest phase space density. Hence the interaction point is defined transversely but has to be reconstructed longitudinally in beam direction. The possibility to adjust the target density along with the gradual consumption of antiprotons for running at constant luminosity is an advantage while the extended interaction-point is a problem for open-charm measurement. Internal cluster targets can run with other gases as well. So not only hydrogen but also nuclear targets will be provided by this target technique. 


\subsubsection{Pellet Target}

Another internal target consists of a stream of frozen hydrogen mini-spheres, called pellets, traversing the antiproton beam perpendicularly. The design will be based on the pellet target placed inside the WASA detector [4] at the CELSIUS storage ring. At the interaction point, typical parameters comprise a pellet rate of $10-15 \mathrm{kHz}$, a pellet speed of of $60 \mathrm{~m} / \mathrm{s}$ and a pellet size of $25-35 \mu \mathrm{m}$. Due to the angular divergence of the pellets, the projected circle area has diameter of about $3 \mathrm{~mm}$, leading to an averaged target thickness of a few $10^{15}$ atoms $/ \mathrm{cm}^{2}$. Since a single pellet undergoes about 100 interactions during the time it traverses the (defocused) beam, it will be possible to determine the position of individual pellets with the necessary accuracy not only in transverse but also longitudinal direction. For PANDA, the present performance of the WASA pellet target needs to be improved. The ongoing further development of the target at a dedicated test area at CELSIUS is expected to improve the present performance in pellet rate and size. Meantime, the production of deuterium pellets has been established, and the use of even heavier targets is to be envisaged.

\subsubsection{Other Targets}

Concerning nuclear targets a technique with fibers or wires may be used. The special targets for hypernuclear physics are described elsewhere [3]. The excellent beam emittance of better than $1 \mathrm{~mm}$ mrad can be expected for the HESR to be exploited best. While problems with beam heating apply also for fiber or wire targets, they pose no additional constraints on the detector system.

\subsubsection{Micro -Vertex Detector}

The design of the micro-vertex detector (MVD) for the target spectrometer is optimized for the detection of secondary vertices and maximum acceptance close to the interaction point. It will also strongly improve the transverse momentum resolution. In addition a layer of diamond or Si detectors may provide a start signal. The concept of the MVD is based on radiation hard silicon pixel detectors with fast individual pixel readout circuits. The layout foresees a five layer barrel detector with an inner radius of $11 \mathrm{~mm}$ and an outer radius of $60 \mathrm{~mm}$. It will have 7.2 million pixels of $50 \mathrm{x} 300 \mu \mathrm{m}^{2}$ size. For optimal vertex resolution in both azimuthal and longitudinal direction, the pixels will be arranged longitudinally for the inner three layers, and azimuthally for the outer two layers. Five detector layers arranged perpendicular to the beam will give best acceptance for the forward part of the particle spectrum. This detector part contains about two million pixels with $100 \mathrm{x} 150 \mu \mathrm{m}^{2}$ size. All detector wafers are $200 \mu \mathrm{m}$ thick $\left(0.25 \% X_{0}\right)$.

The readout via bump-bonded wafers with ASICs as it is used in ATLAS [5] and CMS [6] is foreseen as the default solution. It is highly parallelized and allows zero suppression as well as the transfer of analog information at the same time. The readout wafer has a thickness of $300 \mu \mathrm{m}\left(0.37 \% X_{0}\right)$. An alternative readout scheme is presently in development for future high-energy experiments and has already been used in X-ray astronomy [7]. Active pixels contain the charged particle sensitive part on one side and implement each pixels readout circuit on the other side of the same silicon wafer. The clear advantage 
is that only half of the material thickness has to be used for the same detection capability, which reduces multiple scattering and secondary interactions.

\subsubsection{Tracking Detectors}

The charged-particle tracking devices must handle the high particle fluxes that are anticipated for luminosities of up to $2 \times 10^{32} \mathrm{~cm}^{-2} \mathrm{~s}^{-1}$. The momentum resolution $\Delta p / p$ should typically be on the percent level and the detectors should have a good detection efficiency for secondary vertices which can also occur outside the inner vertex detector This can be achieved by the combination of the vertex detectors, a cylindrical arrangement of straws at large angles, and two sets of 8 (or 6) layer mini drift chambers, similar to those developed for the HADES experiment.

\subsubsection{Straw Tube Tracker}

In total there are 11 double-layers of straws at radial distances between $15 \mathrm{~cm}$ and 42 $\mathrm{cm}$ to the beam and their overall length is $150 \mathrm{~cm}$. The first and last double-layers are arranged parallel to the beam axis, and the remaining 9 double layers are arranged at skew angles ranging from $2^{\circ}$ to $3^{\circ}$. The skew angles cause the stereo layers to form a hyperboloidal shape and allow to determine the position of the particles along the beam direction to about $1 \mathrm{~mm}$. In order to remove the left-right ambiguity the straws are assembled in double-layers which are packed into supports of Al-Be semi-rings. The straws have diameters ranging from $4 \mathrm{~mm}$ (innermost) to $8 \mathrm{~mm}$ (outermost). The tube wall will have a thickness of about $30 \mu \mathrm{m}$ (aluminized mylar, or carbon loaded kapton). As sense wires $20 \mu \mathrm{m}$ thick gold plated tungsten wires are foreseen. The amount of material could be minimized by self-supporting tubes held by a light Al-Be mechanical frame. The gas mixture used will be Argon based with $\mathrm{CO}_{2}$ as quencher. We foresee to have a gas gain not greater than $10^{5}$ in order to guarantee long term operation. With these parameters, we expect a resolution in $x$ and $y$ coordinates of about $150 \mu \mathrm{m}$.

\subsubsection{Mini Drift Chambers}

Particles emitted at angles below $22^{\circ}$ which are not covered fully by the straw chamber will be tracked with two multi-wire drift chambers (MDC) placed $1.4 \mathrm{~m}$ and $2.0 \mathrm{~m}$ downstream of the target. The chambers have to stand a high counting rate of particles peaked at the most forward angles due to the relativistic boost of the reaction products as well as due to the small angle $\bar{p} p$ elastic scattering. With the envisaged luminosity the expected particle flux in the first chamber in the vicinity of the $5 \mathrm{~cm}$ diameter beam pipe is about $2 \times 10^{4} \mathrm{~cm}^{-2} \mathrm{~s}^{-1}$. In addition, the chambers have to work in the $2 \mathrm{~T}$ magnetic field produced by the PANDA-solenoid. In order to fulfill these requirements quadratic (or hexagonal) drift cells with an area of $1 \mathrm{~cm}^{2}$ were chosen. Each chamber will contain several pairs of detection planes. The number of pairs and their orientation will depend upon the actual symmetry (quadratic or hexagonal). The planes in each pair will be staggered by half of the cell width in order to resolve the left-right ambiguity. The chambers will be mounted on a frame of octagonal (or hexagonal) shape. We also explore a possibility of using frames having a form of a closed semi-circle. The detection planes would consist of pairs 
of such frames put together along the common diameter. These frames could be mounted and dismounted without removing the beam-pipe.

\subsubsection{Particle Identification (PID)}

For hadrons and leptons over a large range of solid angle and momenta is an essential requirement for meeting the physics objectives at the HESR. Charged particles in a medium with index of refraction $n$, propagating with velocity $\beta c<1 / n$, emit radiation at an angle $\vartheta_{C}=\arccos (1 / \beta n)$. . Thus, the mass of the detected particle can be determined by combining the velocity information determined from $\vartheta_{C}$ with momentum information from the tracking detectors.

Within the TS, at polar angles between $22^{\circ}-140^{\circ}$ particle identification can be performed by collecting internally reflected Cherenkov (DIRC) light produced inside a glass barrel as realized in the $B a B a r$ detector[8]. It consists of $1.7 \mathrm{~cm}$ thick quartz slabs $(n=1.544)$ surrounding the beam line at a radial distance of $48 \mathrm{~cm}$. The main costs of the BaBar setup arose from the 11000 photomultiplier tubes, which allow timing information to be used to suppress the large background resulting from the electron beam environment. At PANDA only 7000 photomultipliers are necessary. Alternatively, new optical readout schemes may be employed e.g. with APD arrays or by replacing one of the two space dimensions for the Cherenkov rings by the timing information.

For covering the forward section $\left(22^{\circ}\right.$ to $\left.5^{\circ}\right)$ with superior PID a disk shaped DIRC is being developed, which may be located inside the solenoid or, if space does not allow, just outside the magnet yoke. This Disk-DIRC must use focusing optics for read-out with APDs working in Geiger mode. Their quality has to advance for getting suited as photo detectors in this application.

\subsubsection{Muon Detection}

The muon detection in PANDA suffers from the very low cross section of the interesting channels compared to muons from the decay of pions which are abundantly produced in $\bar{p} p$ annihilation. Behind the iron yoke of the target spectrometer, plastic scintillator counters for muon detection will be installed that cover the laboratory polar angular range from $80^{\circ}$ down to the opening for the dipole. Coverage to larger polar angles is not needed since the muons are lower in energy and will be stopped in the iron yoke. The momentum of those muons is determined by the tracking. The muon detection system will consist of about 96 individual bars that are $10 \mathrm{~cm}$ wide, $2 \mathrm{~cm}$ thick and about $2 \mathrm{~m}$ long. In addition there is an equal number of bars perpendicular to the beam axis, at the front of the TS-solenoid. The bars are read out with photomultipliers fed to ADCs and TDCs. This information can be used to determine the position for matching with the TS tracking, and the time of flight, to suppress random coincidences. The energy information provides not only a redundant position information, but also a value of $d E / d x$ to permit further background suppression. Alternatively, mini drift tubes (MDT), which are used at the D0-detector of Fermilab and at COMPASS of CERN may replace the plastic scintillators. They allow for much improved spatial resolution however at the expense of much higher costs 


\subsubsection{Electromagnetic calorimeter}

Expected high count rates and a geometrically compact design of the target spectrometer require a fast scintillator material with a short radiation length and Moliére radius for the construction of the electromagnetic calorimeter (Ecal). In recent years $\mathrm{PbWO}_{4}$ has been proposed and confirmed as a high density inorganic scintillator with sufficient energy and time resolution for photon, electron and hadron detection even at intermediate energies For high energy physics $\mathrm{PbWO}_{4}$ has been chosen by the CMS and ALICE collaborations at CERN and optimized for large scale production. Besides a short decay time of less than 10 ns good radiation hardness has been achieved [9]. Recent developments indicate a significant increase of light yield due to crystal perfection and appropriate doping to enable photon detection down to $10 \mathrm{MeV}$ with sufficient resolution. Cooling down to $-25^{\circ} \mathrm{C}$ will further increase the light yield by a factor 3 compared to room temperature operation. It is proposed to use crystals of approximately $20 X_{0}$ in length achieving an energy resolution below $2 \%$ at $1 \mathrm{GeV}$ at a tolerable energy loss due to longitudinal leakage of the shower. These crystals allow $\pi$-e discrimination of $10^{3}$ for momenta above $0.5 \mathrm{GeV} / \mathrm{c}$. Therefore, particle identification does not rely on an additional gas Cherenkov detector in favor of a very compact geometry. Tapered crystals with a front size of $2 \times 2 \mathrm{~cm}^{2}$ mounted with an inner radius of $53 \mathrm{~cm}$ will be used. This implies 11000 crystals for the barrel part of the calorimeter. The backward and forward end-caps will comprise additional 30005000 modules. The readout of the crystals will be accomplished by large area avalanche photodiodes with optimized light collection, a tolerable nuclear counter effect and a fast timing information.

\subsubsection{Target Spectrometer Solenoid}

The target solenoid consists of a superconducting coil with an inner radius of $0.95 \mathrm{~m}$ and a length of $3.0 \mathrm{~m}$. The maximum magnetic field is $2 \mathrm{~T}$. The field homogeneity is better than $2 \%$ over the volume of the straw tracker. In order to minimize the amount of material in front of the calorimeter, the latter is placed inside the magnetic coil. The bending power downstream of the target allows a reasonable momentum resolution even at the smallest polar angles of $5^{\circ}$ of the target spectrometer. This minimum angle of the solenoid acceptance is small enough to confine the dipole magnet to a $1 \mathrm{~m}$ gap height. The cryostat for the coils has two warm bores of $100 \mathrm{~mm}$ diameter one on top and the other on bottom of the target position to allow for insertion of internal targets.

\subsection{Forward spectrometer}

\subsubsection{Dipole Magnet}

A normal conducting dipole magnet of window frame type with $1 \mathrm{~m}$ gap and $2 \mathrm{~m}$ aperture will be used for the momentum analysis of charged particles in the forward spectrometer (FS) The magnet yoke will occupy the space between $4 \mathrm{~m}$ and $6 \mathrm{~m}$ downstream of the target and will define the angular acceptance of the FS to $\pm 10^{\circ}$ in the horizontal direction and $\pm 5^{\circ}$ in the vertical direction. The maximum bending power of the magnet will be $2 \mathrm{Tm}$ and the resulting deflection of the antiproton beam at the maximum momentum 
of $15 \mathrm{GeV} / \mathrm{c}$ will be $2.2^{\circ}$. The iron yoke of this dipole magnet can be instrumented with detectors for ionizing particles. This option will become important for maximizing the muon detection efficiency. The deflection of the antiproton beam will be compensated by three back-bending dipole magnets placed downstream of the PANDA detector (chicane).

\subsubsection{Tracking Detectors}

The deflection of particle trajectories in the field of the dipole magnet will be measured with a set of 4 drift chambers (MDC), two placed before and two behind the dipole magnet. The chambers will have rectangular frames with an active area of $1.3 \times 0.7 \mathrm{~m}^{2}$ in the first chamber and $2.4 \times 1.2 \mathrm{~m}^{2}$ in the second pair. The chambers will contain quadratic (or hexagonal) drift cells of $1 \mathrm{~cm}$ width, the same as in the TS-chambers. Each chamber will contain three pairs of detection planes: one pair with vertical wires and two pairs with wires inclined by $+45^{\circ}$ and $45^{\circ}$ (or slightly smaller). This configuration will allow to reconstruct tracks in each chamber separately also in case of multi-track events. The beam-pipe will pass through central holes in the chambers and the most central wires will be either eliminated or mounted on additional insulating rings surrounding the pipe. The expected momentum resolution of the system for $3 \mathrm{GeV} / \mathrm{c}$ protons is $\Delta p / p=0.2 \%$ and is limited mainly by the small angle scattering on the chamber wires and gas.

\subsubsection{Time-of-Flight Detector}

The stop time-of-flight detector (TOF) will consist of a wall of tiles made of organic scintillator and read out on both ends by fast photomultipliers. The TOF will be measured between the start detector placed in the target region and the stop wall placed about $7 \mathrm{~m}$ from the target. With the expected time resolution of $\sigma=100 \mathrm{ps} \pi-K$ and $K-p$ separation on a $3 \sigma$ level will be possible up to momenta of $2.8 \mathrm{GeV} / c$ and $4.7 \mathrm{GeV} / c$, respectively. $\mathrm{R} \& \mathrm{D}$ is going on at St. Petersburg(PNPI) for improving time resolution using selected scintiallators and photomultipliers [10].

\subsubsection{Ring Imaging Cherenkov Counter}

In order to extend the momentum range for the $\pi-K$ and $K$-p separation beyond that of the TOF system we consider to use a dual-radiator RICH detector like the one developed for the HERMES experiment [11]. Using two radiators silica aerogel and $\mathrm{C}_{4} \mathrm{~F}_{10}$ gas with different indices of refraction equal to 1.0304 and 1.00137 , respectively, provides $\pi$ $-K-p$ separation in a broad momentum range from $2-15 \mathrm{GeV} / c$. Due to the usage of a lightweight mirror which focuses the Cherenkov light on an array of photomultipliers placed outside the active volume, the total thickness of the detector is reduced to the freon gas radiator $\left(5 \% X_{0}\right)$, the aerogel radiator $\left(2.8 \% X_{0}\right)$ and the aluminum window $\left(3 \% X_{0}\right)$.

\subsubsection{Calorimeter and Muon Detection}

As a calorimeter for the forward region we consider to use the mid-rapidity calorimeter MIRAC from the WA80 experiment. MIRAC consists of 30 independent stacks with the dimensions of $22 \mathrm{~cm}$ horizontally and $120 \mathrm{~cm}$ vertically. Each stack consists 
of a lead-scintillator electromagnetic section $15 X_{0}$ long, and a steel-scintillator hadronic section $6 X_{0}$ long. The energy resolution for electromagnetic and hadronic showers is $(0.014+0.11 / \sqrt{E / G e V}) \%$ and $(0.034+0.34 / \sqrt{E / G e V}) \%$, respectively. Since the energy resolution of the electromagnetic section is rather poor, we consider to remove this part from MIRAC and to replace it by Shashlyk-type calorimeter modules [12] which will achieve a much better resolution. This calorimeter will make it possible to measure besides energies of electrons and photons also energies of neutrons and anti-neutrons which are not registered in other components of the FS. Finally, behind the FS-calorimeter and the yoke of the dipole magnet there will be a wall of counters for muon identification similar to the ones in the TS (plastic scintillators or mini drift tubes).

\section{Trigger and electronics}

In many contemporary experiments the trigger and data acquisition (DAC) system is based on a two layer hierarchical approach. A subset of especially instrumented detectors is used to evaluate a first level trigger condition. For the accepted events, the full information of all detectors is then transported to the next higher trigger level or to storage. The time available for the first level decision is usually limited by the buffering capabilities of the frontend electronics. Furthermore, the hard-wired detector connectivity severely constrains both the complexity and the flexibility of the possible trigger schemes. We aim at the development of a data acquisition concept which is much better matched to the high data rates and to the complexity of the next generation of experiments. In our approach all detector channels are self triggering entities. They autonomously detect signals and pre-process them to extract and transmit only the physically relevant information. The data related to a particle hit, substantially reduced in the pre-processing step, is marked by a precise time stamp and buffered for further processing. The trigger selection finally occurs in so called compute nodes which access the buffers via a high bandwidth network fabric. The new concept provides a high degree of flexibility in the choice of trigger algorithms. It makes trigger conditions available which definitely are outside the capabilities of the standard approach, an obvious example being displaced vertex triggering. In addition, all sub-detectors can contribute to the trigger decision on the same footing, and there are no restrictions due to hard-wired connectivity. Different physics can be accessed either in parallel or after software reconfiguration of the system.

\section{Summary}

At present there is development going on in all detector species described here. Some of schemes may still be revised, as for example the straw chamber could be replaced by a time projection chamber. Alternatively, straw chambers may replace the mini drift chambers in the forward spectrometer. Target development is very actively pursued at various laboratories. Magnet design is under way. Prototype modules for the $\mathrm{PbWO}_{4}$ calorimeter are planned and will be built within the next year. There is an enthusiastic collaboration of people [3] from 47 institutions around the world who want to start the 
data taking for this challenging physics program with PANDA in the beginning of the second decade of the twenty-first century.

\section{References}

[1] An International Accelerator facility for Beams of Ions and Antiprotons, Conceptual design report, GSI, 2001

[2] Letter of Intent for PANDA, GSI, 2004

[3] Technical Progress Report for PANDA, GSI, 2005

[4] C. Ekstöm et al,NIM A 371 (1996) 572

[5] The Atlas Collaboration, ATLAS TDR 11, CERN/LHCC 98-13

[6] The CMS Collaboration, CMS TDR 5, CERN/LHCC 98-6

[7] P. Holl et al., IEEE Trans. Nucl. Sc. Vol 47 (2000) 1421

[8] R. Aleksan et al., Nucl. Instr. and Meth. in Phys. Res. A397 (1997) 261

[9] E. Auffray et al., SCINT99, Proceedings, Moscow, 1999

[10] S.L. Belostotski, private communication

[11] N. Akopov et al.NIM in Phys. Res. A 479 (2002) 511

[12] G.S. Atoyan et al. NIM A320, (1992)144 and NIM A531 (2004) 467 
